\title{
Amyloid Beta Peptide 1-42 Induces SH-SY5Y Cell Apoptosis via the Promotion of Meg3 Long Noncoding RNA Expression
}

\author{
Yongyi Huang ${ }^{a-c}$ Te Liu ${ }^{a, b}$ \\ aShanghai Geriatric Institute of Chinese Medicine, Longhua Hospital, Shanghai University \\ of Traditional Chinese Medicine, bShanghai Tenth People's Hospital, Medical School, Tongji \\ University, and 'Shanghai Topbiox Co. Ltd., Shanghai, China
}

Key Words

Alzheimer's disease · Amyloid beta peptide 1-42 · Long noncoding RNAs · Meg3 · p53 ·

Apoptosis

\begin{abstract}
Background/Aim: The mechanisms and processes of amyloid beta $(A \beta)_{1-42} / A \beta_{1-40}$ degeneration and deposition of neuron damage are still not clear. The long noncoding RNA (IncRNA) is one of the members of the noncoding RNA family. In this study, we aimed to investigate whether $A \beta_{1-42}$ inhibited SH-SY5Y cells in vitro through modulating Meg3 IncRNA. Methods: The Alzheimer's disease (AD) senile plaque cell model was generated using synthetic $A \beta_{1-42^{-}}$ treated SH-SY5Y cells. MTT assays were used to determine the proliferation of SH-SY5Y cells. Quantitative (q)RT-PCR and Western blot analyses were used to test the expression levels of mRNA and protein. Northern blot analysis was used to confirm Meg3 IncRNA expression. Results: The MTT assays showed that exogenous $A \beta_{1-42}$ suppressed SH-SY5Y cells. The qRT-PCR and Western blot analyses revealed that the expression of p53 mRNA and protein was significantly increased in the AD model group, with a marked decrease in MDM2 and Ki-67 expression on day 7. Moreover, the qRT-PCR and Northern blot analyses confirmed that exogenous $A \beta_{1-42}$ promoted the expression of Meg3 IncRNA. There was a downregulation of Meg3 IncRNA expression in SH-SY5Y cells by siRNA, which could promote of the ability of MDM2 to degrade p53 protein on the ubiquitin pathway and delay SH-SY5Y apoptosis. Conclusion: Meg3 IncRNA is implicated as an important factor in the formation of mature A $\beta$ peptides.
\end{abstract}




\section{Introduction}

The occurrence of Alzheimer's disease (AD) is closely related to extracellular amyloid beta $(A \beta)_{1-42} / A \beta_{1-40}$ deposits and intracellular hyperphosphorylated tau protein aggregates $[1,2]$. However, the mechanisms and processes of $A \beta_{1-42} / A \beta_{1-40}$ degeneration and deposition of neuron damage are still not clear. The long noncoding RNA (lncRNA) is one of the members of the noncoding RNA family. It is a long RNA molecule that contains over 200 nucleotides without the ability to encode proteins and is widely involved in the regulation of many important cellular functions, including the transcriptional regulation of gene expression, the developmental regulation of tissues and cells inside the body, the regulation of stem cell pluripotency and the reprogramming of somatic cells, and the regulation of the occurrence and development of the disease. The human maternally expressed gene 3 (Meg3) is an mRNA-like noncoding RNA with a nucleotide length of $>1.6 \mathrm{~kb}$ [3-5]. It is a paternally imprinted gene which lies on human chromosome 14q. The Meg3 gene consists of 10 exons which form some small open reading frames by alternative splicing. However, these open reading frames do not resemble any known functional proteins or peptides. Previous studies showed that Meg3 was highly expressed in many normal human tissues, especially in the brain and pituitary gland [3-5]. Meanwhile, they have confirmed that human Meg3 is an IncRNA [3, 4]. Zhou and colleagues $[3,4]$ showed that Meg3 lncRNA could suppress tumor proliferation by activing p53-dependent and -independent pathways. However, the mechanism of Meg3 lncRNA in the occurrence of $\mathrm{AD}$ induced by $\mathrm{A} \beta_{1-42}$ deposition is not clear.

In this study, an $A D$ senile plaque cell model was generated using synthetic $A \beta_{1-42}$-treated SH-SY5Y cells. We aimed to investigate whether $A \beta_{1-42}$ inhibited SH-SY5Y cells in vitro via modulation of Meg3 IncRNA.

\section{Materials and Methods}

\section{Cell Culture and $A \beta_{1-42}$ Treatment}

An AD senile plaque cell model was generated as previously described [6, 7]. The SH-SY5Y cell lines were seeded in a 6-well plate in DMEM supplemented with $10 \%$ fetal calf serum (Invitrogen, Life Technologies Corporation, Grand Island, N.Y., USA), penicillin (100 U/ml), and glutamine $(0.3 \mathrm{mg} / \mathrm{ml})$ and incubated in a humidified tissue culture incubator containing $5 \% \mathrm{CO}_{2}$ at $37^{\circ} \mathrm{C}$ until $80 \%$ confluent. Then, $10 \mu \mathrm{mol} / \mathrm{l}$ of large aggregates of synthetic $\mathrm{A} \beta_{1-42}$ (Sigma-Aldrich, St. Louis, Mo., USA) was added to the cultures. After $24 \mathrm{~h}$, the drug-containing medium was replaced with fresh normal cell medium for continued culture.

\section{MTT Assay for Cell Proliferation}

According to the previously described procedure [8], briefly, each group of SH-SY5Y cells was seeded at $2 \times 10^{3}$ per well in 96-well plates until $85 \%$ confluent. MTT (Sigma-Aldrich) reagent $(5 \mathrm{mg} / \mathrm{ml})$ was added to the maintenance cell medium at different time points and incubated at $37^{\circ} \mathrm{C}$ for an additional $4 \mathrm{~h}$. The reaction was terminated with $150 \mu$ ldimethyl sulfoxide (DMSO; Sigma-Aldrich) per well, and the cells were lysed for $15 \mathrm{~min}$, after which the plates were gently shaken for 5 min. Absorbance values were determined by using the enzyme-linked immunosorbent assay reader (Model 680; Bio-Rad, Hercules, Calif., USA) at $490 \mathrm{~nm}$.

RNA Extraction and Analysis by Quantitative Real-Time PCR

According to the manufacturer's protocol, total RNA from each cell was isolated with TRIzol reagent (Invitrogen). The RNA samples were treated with DNase I (Sigma-Aldrich), 
quantified, and reverse transcribed into cDNA with the ReverTra Ace- $\alpha$ First Strand cDNA Synthesis Kit [Toyobo (Shanghai) Biotech Co., Ltd., Shanghai, China]. Quantitative real-time PCR was conducted with a RealPlex4 real-time PCR detection system from Eppendorf (Hamburg, Germany), with SYBR Green Real-Time PCR Master Mix (Toyobo) as the detection dye. Quantitative real-time PCR amplification was performed over 40 cycles with denaturation at $95^{\circ} \mathrm{C}$ for $15 \mathrm{~s}$ and annealing at $57^{\circ} \mathrm{C}$ for $45 \mathrm{~s}$. Target cDNA was quantified with the relative quantification method. A comparative threshold cycle (Ct) was used to determine gene expression relative to a control (calibrator), and steady-state mRNA levels are reported as an n-fold difference relative to the calibrator. For each sample, the maker gene $\mathrm{Ct}$ values were normalized with the formula $\Delta \mathrm{Ct}=\mathrm{Ct}$ _genes $-\mathrm{Ct} \_$18S RNA. To determine relative expression levels, the following formula was used: $\Delta \Delta \mathrm{Ct}=\Delta \mathrm{Ct}$ _sample groups $-\Delta \mathrm{Ct}$ _control group. The values used to plot relative expressions of markers were calculated with the expression $2^{-\Delta \Delta \mathrm{Ct}}$. The mRNA levels were calibrated on the basis of levels of $18 \mathrm{~S}$ rRNA. The cDNA of each gene was amplified with primers as previously described $[3,8]$.

\section{Western Blot Analysis}

According to the previously described procedure [6], cells were lysed using a $2 \times$ loading lysis buffer (Beyotime Institute of Biotechnology, Shanghai, China). The total amount of protein from the cultured cells was subjected to 12\% SDS-PAGE and transferred onto HybridPVDF membranes (Millipore, Bedford, Mass., USA). After blocking with 5\% (w/v) nonfat dried milk in TBST (Beyotime), the PVDF membranes were washed 4 times (15 min each) with TBST at room temperature and incubated with primary antibody. Following extensive washing, the membranes were incubated with HRP-conjugated goat anti-rabbit IgG secondary antibody (1:1,000; Santa Cruz Technology, Santa Cruz, Calif., USA) for $1 \mathrm{~h}$. After washing 4 times (15 min each) with TBST at room temperature, the immunoreactivity was visualized by enhanced chemiluminescence using the ECL kit from PerkinElmer Life Sciences (Norwalk, Conn., USA).

\section{RNA Extraction and Northern Blot Analysis}

All steps of Northern blotting were conducted according to the previously described procedure [3, 8]. For all groups, $20 \mu \mathrm{g}$ of good-quality total RNA was analyzed on a $7.5 \mathrm{M}$ urea $12 \%$ PAA denaturing gel and transferred to a Hybond $\mathrm{N}^{+}$nylon membrane (Amersham, Freiburg, Germany). Membranes were cross-linked using UV light for $30 \mathrm{~s}$ at $1,200 \mathrm{~mJ} / \mathrm{cm}^{2}$. Hybridization was performed with an antisense StarFire probe to detect Meg3 lncRNA fragments according to the instructions of the manufacturer [3]. After washing, the membranes were exposed for 20-40 h to Kodak XAR-5 films (Sigma-Aldrich). As a positive control, all membranes were hybridized with a human U6 snRNA probe: 5'-GCAGGGGCCATGCTAATCTTCTCTGTATCG-3'. Exposure times for the U6 control probe varied between 15 and $30 \mathrm{~min}$.

\section{SiRNA and Cell Transfection}

A siRNA-targeted Meg3 IncRNA expression plasmid was constructed as previously described [3]. SH-SY5Y cells were transfected with $0.3 \mu$ g siRNA-Meg3 or siRNA-mock vector using Lipofectamine 2000 (Invitrogen) according to the manufacturer's protocol.

\section{Statistical Analysis}

Each experiment was performed as least 3 times. The data are shown as means \pm SE. Differences were evaluated using Student's t test. A probability of $<0.05$ was considered to be statistically significant. 
Huang and Liu: Amyloid Beta Peptide 1-42 Induces SH-SY5Y Cell Apoptosis via the

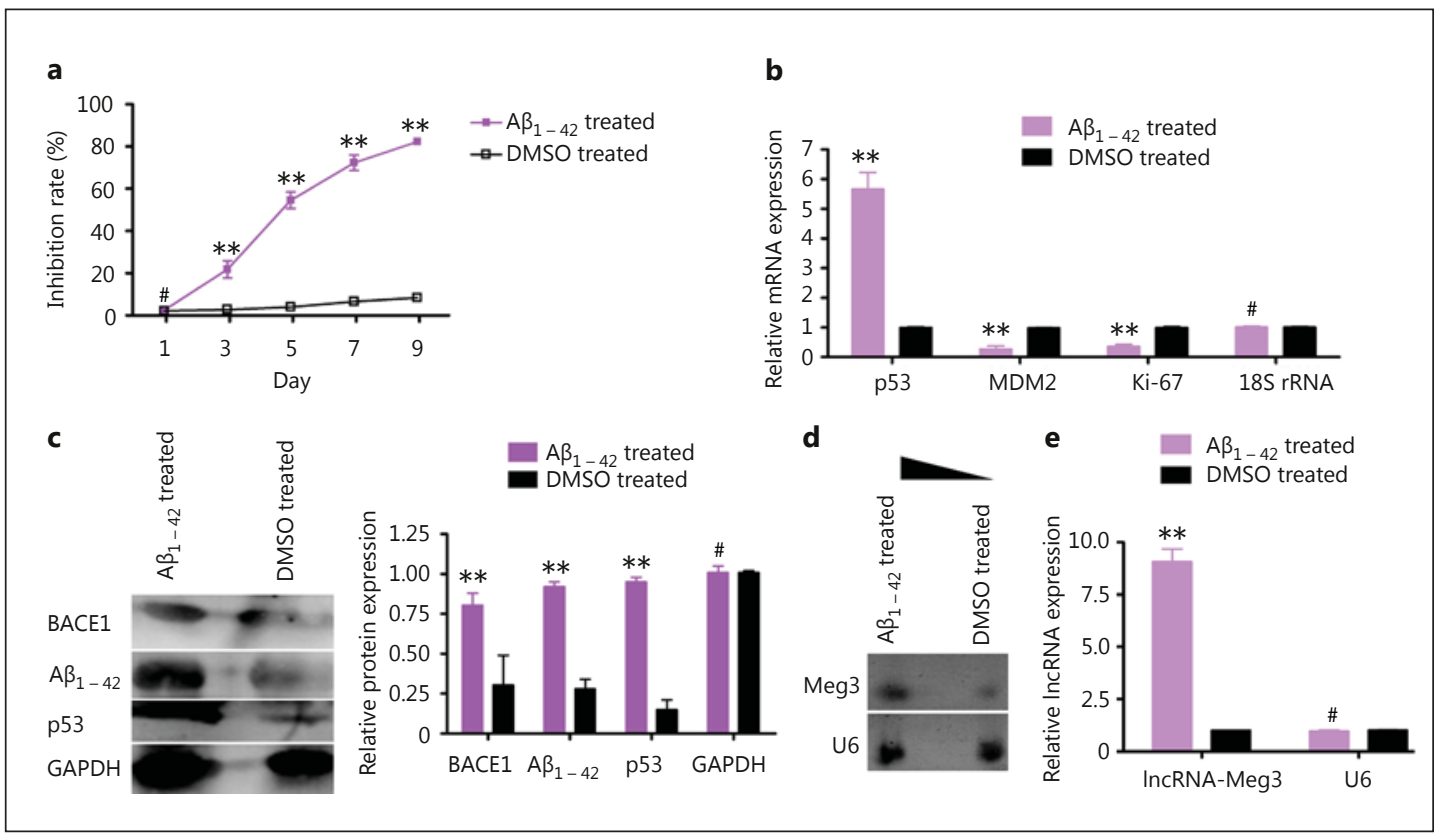

Fig. 1. Exogenous $A \beta_{1-42}$ influenced SH-SY5Y cell proliferation and Meg3 lncRNA expression. a The MTT assays showed that large aggregates of synthetic $A \beta_{1-42}$ inhibited SH-SY5Y cell proliferation in a time-dependent manner. $\mathbf{b}$ The results of qRT-PCR showed that the mRNA expression of p53 in the A $\beta_{1-42}$ treatment group was elevated markedly, while MDM2 and Ki-67 expression (normalized against 18S rRNA levels) in this group was markedly decreased compared with that in the DMSO-treated group on day 7. c Western blotting confirmed that expression of the BACE1, p53, and endogenous $A \beta_{1-42}$ proteins was significantly increased in the $A \beta_{1-42}$ treatment group compared with the DMSO-treated group on day 7. GAPDH was used as a loading control. d Northern blotting showed that the Meg3 lncRNA hybridization signals were higher in A $\beta_{1-42}$-treated cell extracts than in the DMSO-treated group. e The results of qRT-PCR showed that Meg3 IncRNA expression in the $A \beta_{1-42}$ treatment group was elevated markedly (normalized against U6 RNA levels) compared with that in the DMSO-treated group on day 7. ${ }^{* *} \mathrm{p}<0.01,{ }^{*} \mathrm{p}>0.05$ vs. DMSO-treated group $(\mathrm{n}=3)$.

Table 1. Results of the MTT assays (\%)

\begin{tabular}{lrlll}
\hline Day & $\mathrm{A} \beta_{1-42}$ treated & DMSO treated & \multicolumn{2}{c}{$\mathrm{A} \beta_{1-42}$ treated, \% } \\
\cline { 3 - 5 } & & & siRNA-Meg3 & \multicolumn{1}{c}{ siRNA-mock } \\
\hline 1 & $2.537 \pm 0.782$ & $2.177 \pm 0.780$ & $7.730 \pm 0.746$ & $10.433 \pm 1.133$ \\
3 & $21.890 \pm 4.062$ & $2.780 \pm 0.995$ & $13.343 \pm 2.073$ & $25.697 \pm 2.546$ \\
5 & $54.573 \pm 3.944$ & $4.007 \pm 0.529$ & $29.947 \pm 1.442$ & $52.720 \pm 3.111$ \\
7 & $72.300 \pm 3.632$ & $6.640 \pm 0.290$ & - & - \\
9 & $82.257 \pm 1.732$ & $8.497 \pm 1.261$ & - & - \\
\hline
\end{tabular}

\section{Results}

Exogenous $A \beta_{1-42}$ Suppressed SH-SY5Y Cell Proliferation and Induced High Meg3 IncRNA Expression

The results of the MTT assays showed that large aggregates of synthetic $A \beta_{1-42}$ suppressed the proliferation of SH-SY5Y cells in a time-dependent manner (fig. 1a; table 1). At the same time, the results of quantitative (q)RT-PCR showed that the expression of p53 in the A $\beta_{1-42^{-}}$ 
a

$\rightarrow A \beta_{1-42}$ treated + siRNA-Meg3

$\rightarrow A \beta_{1-42}$ treated + siRNA-mock

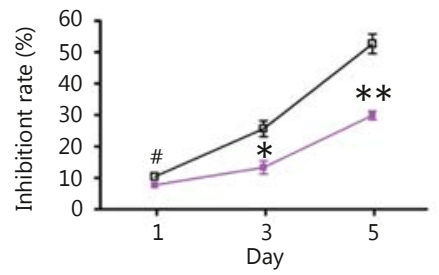

C

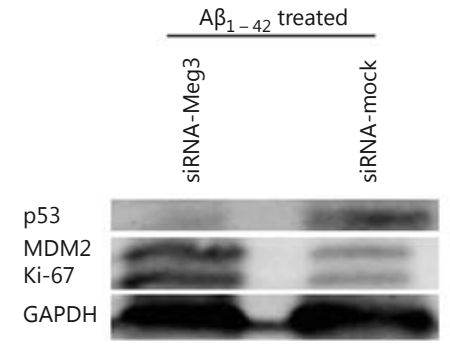

b $\quad \begin{aligned} & A \beta_{1-42} \text { treated + siRNA-Meg3 } \\ & A \beta_{1-42} \text { treated + siRNA-mock }\end{aligned}$
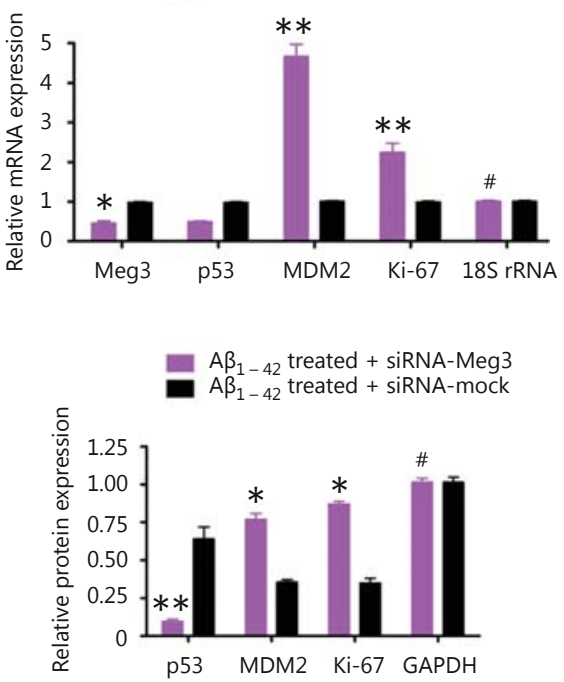

Fig. 2. Attenuation of the cytotoxicity of exogenous $A \beta_{1-42}$ by siRNA suppression of Meg3 IncRNA expression. a The MTT assays showed that the inhibition rates of the siRNA-Meg3-transfected SH-SY5Y cell group were decreased significantly compared with those of the siRNA-mock-transfected group on days 3 and 5 after treatment with $A \beta_{1-42}$. b The results of qRT-PCR showed that the mRNA expression of MDM2 and Ki-67 in the siRNA-Meg3-transfected SH-SY5Y cell group was elevated markedly, while Meg3 lncRNA and p53 expression (normalized against 18S rRNA levels) in this group was markedly decreased compared with that in the siRNA-mock-transfected group on days 3 and 5 after treatment with $A \beta_{1-42}$ on day 5. c Western blotting confirmed that the expression of the Ki-67 and MDM2 proteins was significantly increased in the siRNA-Meg3transfected SH-SY5Y cell group compared with the siRNA-mock-transfected group after treatment with A $\beta_{1-}$ 42 on day 5 . However, the expression of p53 protein was significantly decreased in the siRNA-Meg3-transfected group compared with the siRNA-mock-transfected group after treatment with $A \beta_{1-42}$ on day 5. GAPDH was used as a loading control. ${ }^{* *} \mathrm{p}<0.01,{ }^{*} \mathrm{p}<0.05,{ }^{*} \mathrm{p}>0.05$ vs. siRNA-mock group $(\mathrm{n}=3)$.

treated group was markedly elevated compared with that in the DMSO-treated control group, while MDM2 and Ki-67 expression was decreased on day 5 of $A \beta_{1-42}$ treatment (fig. 1b). Moreover, Western blotting confirmed that expression levels of $\mathrm{p} 53, \mathrm{~A} \beta_{1-42}$, and BACE1 were significantly increased in the $A \beta_{1-42}$-treated group compared with the control group (fig. 1c). In addition, Northern blot analysis indicated that Meg3 lncRNA hybridization signals were higher in the $A \beta_{1-42}$-treated cell extracts than in the control group (fig. $1 \mathrm{~d}$ ). The results of qRT-PCR also showed that the expression of Meg3 lncRNA in the $A \beta_{1-42}$-treated group was elevated markedly compared with that in the DMSO-treated control group (fig. 1e). These data indicate that exogenous $A \beta_{1-42}$ not only inhibited SH-SY5Y cell proliferation and induced the expression of amyloid precursor protein-related factors, but also promoted Meg3 lncRNA expression.

\section{Attenuation of the Ability of $A \beta_{1-42}$ by siRNA Silencing of Meg3 IncRNA Expression}

The results of the MTT assays showed that the inhibition rates of the siRNA-Meg3-transfected SH-SY5Y cell group were decreased significantly in relation to those of the siRNAmock-transfected group on days 3 and 5 after treatment with $A \beta_{1-42}$ (fig. 2a; table 1). Moreover, the results of qRT-PCR showed that the expression of Meg3 lncRNA and p53 in the siRNA-Meg3-transfected group was markedly decreased, while MDM2 and Ki-67 expression 
Huang and Liu: Amyloid Beta Peptide 1-42 Induces SH-SY5Y Cell Apoptosis via the Promotion of Meg3 Long Noncoding RNA Expression

Fig. 3. The pathway of Meg3 lncRNA regulates MDM2 and p53 protein degradation. $\mathrm{Ub}=$ Ubiquitin.

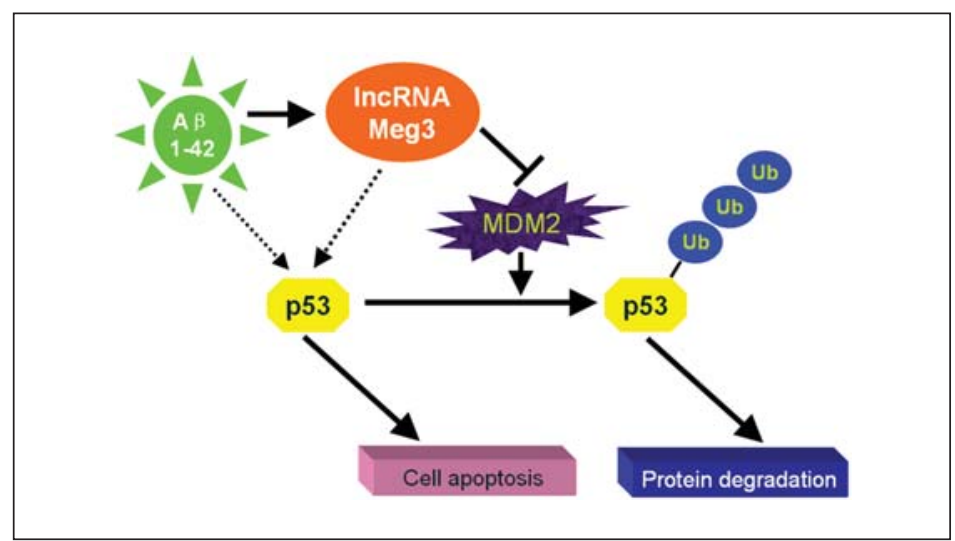

was markedly elevated compared with that in the siRNA-mock-transfected group on day 5 after treatment with $A \beta_{1-42}$ (fig. 2b). Meanwhile, Western blotting revealed that the expression levels of MDM2 and Ki-67, but not those of p53, were significantly increased in the siRNAmock-transfected group on day 5 after treatment with $A \beta_{1-42}$ (fig. 2c). These results indicate that the cytotoxicity of $A \beta_{1-42}$ was weakened when Meg3 IncRNA and p53 protein were expressed to a lesser extent.

\section{Discussion}

AD is a complex progressive neurodegenerative disease that is characterized by an irreversible cognitive functional decline, a loss of memory, and a high degree of heterogeneity in clinical parameters. It has become the third most lethal disease in the world [1, 2]. IncRNAs are fine-tuning modifiers of nervous system developmental regulation, but knowledge regarding their control of $\mathrm{AD}$ is lacking [9]. In a study of the pathogenesis of AD, Faghihi et al. [10] identified an IncRNA that acted as a positive regulator of its target gene. They identified an antisense $\beta$-secretase (BACE1-AS) IncRNA which generates A $\beta$. This IncRNA increased the stability of the BACE1 mRNA, thus leading to an amplified production of $A \beta$ peptides and the deleterious feed-forward cycles of disease progression [10].

In our previous study, we showed that downregulation of BACE1-AS IncRNA expression in SH-SY5Y cells by siRNA silencing resulted in attenuation of the ability of BACE1 to cleave amyloid precursor protein and delayed the induction of senile plaque formation in the AD senile plaque SH-SY5Y cell model [8]. In the present study, we demonstrated that exogenous synthetic $A \beta_{1-42}$ could significantly promote the expression of Meg3 lncRNA, p53, endogenous BACE1, and $A \beta_{1-42}$ in SH-SY5Y cells and significantly weakened the expression of MDM2 and Ki-67. However, p53 and Meg3 lncRNA expression was significantly reduced in SH-SY5Y cells as a result of the siRNA-mediated silencing of Meg3 IncRNA expression. Furthermore, exogenous $A \beta_{1-42}$ did not stimulate the formation of endogenous $A \beta_{1-42}$ in siRNA-Meg3-transfected SH-SY5Y cells.

These data indicate that inhibition of Meg3 lncRNA expression effectively inhibits the endogenous production of $A \beta$ peptides. In contrast, when the expression of Meg3 lncRNA siRNA was silenced in transfected SH-SY5Y cells treated with exogenous A $\beta$ peptides, the cytotoxic effects of $A \beta$ were significantly reduced and these cells maintained their normal state. In addition, MDM2, one of the E3 ubiquitin ligases, is a direct p53 transcriptional target and also the most critical negative regulator of p53 $[11,12]$. It can form an autoregulatory 
negative feedback loop with p53 in the cell to tightly regulate the levels and activity of p53. Also, it has been shown that MDM2 is an oncogene, and it was identified as an inhibitor of DNA break repair $[11,12]$. In our study, we identified exogenous $A \beta_{1-42}$-stimulated Meg3 IncRNA as a new regulator that directly repressed MDM2 to activate p53 and enhance p53 function in SH-SY5Y cells (fig. 3). Thus, Meg3 lncRNA is implicated as an important factor in the formation of mature $A \beta$ peptides. The ability of MDM2 to degrade p53 protein on the ubiquitin pathway was elevated via silencing of Meg3 lncRNA expression.

\section{Acknowledgements}

This work was supported by a grant from the National Natural Science Foundation of China (No. 81202811) and funded by the China Postdoctoral Science Foundation (No. 2014M550250, 2015T80455) and the Shanghai Municipal Health Bureau Fund (No. 20124320) to T. Liu.

\section{Disclosure Statement}

We declare no potential conflicts of interest.

\section{References}

1 Querfurth HW, LaFerla FM: Alzheimer's disease. N Engl J Med 2010;362:329-344.

-2 Lu H, Zhu XC, Jiang T, Yu JT, Tan L: Body fluid biomarkers in Alzheimer's disease. Ann Transl Med 2015;3:70.

3 Zhou Y, Zhong Y, Wang Y, et al: Activation of p53 by MEG3 non-coding RNA. J Biol Chem 2007;282:2473124742.

4 Zhou Y, Zhang X, Klibanski A: MEG3 noncoding RNA: a tumor suppressor. J Mol Endocrinol 2012;48:R45-R53.

5 Miyoshi N, Wagatsuma H, Wakana S, et al: Identification of an imprinted gene, Meg3/Gtl2 and its human homologue MEG3, first mapped on mouse distal chromosome 12 and human chromosome 14q. Genes Cells 2000; 5:211-220.

6 Liu T, Shen D, Xing S, et al: Attenuation of exogenous angiotensin II stress-induced damage and apoptosis in human vascular endothelial cells via microRNA-155 expression. Int J Mol Med 2012;31:188-196.

7 Isobe I, Yanagisawa K, Michikawa M: A possible model of senile plaques using synthetic amyloid $\beta$-protein and rat glial culture. Exp Neurol 2000;162:51-60.

-8 Liu T, Huang Y, Chen J, et al: Attenuated ability of BACE1 to cleave the amyloid precursor protein via silencing long noncoding RNA BACE1AS expression. Mol Med Rep 2014;10:1275-1281.

$\checkmark 9$ Wilusz JE, Sunwoo H, Spector DL: Long noncoding RNAs: functional surprises from the RNA world. Genes Dev 2009;23:1494-1504.

10 Faghihi MA, Modarresi F, Khalil AM, et al: Expression of a noncoding RNA is elevated in Alzheimer's disease and drives rapid feed-forward regulation of $\beta$-secretase. Nat Med 2008;14:723-730.

11 Zhang C, Liu J, Wang X, Feng Z: The regulation of the p53/MDM2 feedback loop by microRNAs. RNA Dis 2015; 2:e502.

12 Carrillo AM, Hicks M, Khabele D, Eischen CM: Pharmacologically increasing Mdm2 inhibits DNA repair and cooperates with genotoxic agents to kill p53 inactivated ovarian cancer cells. Mol Cancer Res 2015;13:11971205. 